\title{
Shear-wave splitting of Sichuan Regional Seismic Network*
}

\author{
ZHANG Yong-jiu ${ }^{1), *}$ (张永久) GAO Yuan ${ }^{2)}$ (高 原) SHI Yu-tao ${ }^{2)}$ (石玉涛) \\ CHENG Wan-zheng ${ }^{1)}$ (程万正) \\ 1) Earthquake Administration of Sichuan Province, Chengdu 610041, China \\ 2) Institute of Earthquake Science, China Earthquake Administration, Beijing 100036, China
}

\begin{abstract}
Using seismic data recorded by the Chengdu Digital Seismic Network from May 1, 2000 to December 31, 2006, we obtain the dominant polarization directions of fast shear-waves at eight digital seismic stations adopting the SAM technique. The results show that the dominant directions of polarizations of fast shear-waves at most of stations are mainly in nearly NE-SW or NW-SE direction in Sichuan. The dominant polarization directions of the fast shear-waves at stations located at the active faults or intersection of several active faults are consistent with the strikes of active faults which control the earthquakes used in the analysis, and are basically consistent with the directions of regional compression axis. However, several stations show that the fast shear-waves are not consistent with the strikes of active faults and the directions of regional compression axis, due to the influence of local complicated crustal structure.
\end{abstract}

Key words: shear-wave splitting; polarization of fast shear-wave; compression axis; active fault; Sichuan region

CLC number: P135. $3^{+} 1 \quad$ Document code: A

\section{Introduction}

Anisotropy of the crust is a common phenomenon (Crampin, 1984), shear-wave splitting can be used to study the earthquake anisotropic characteristic in crust, to analyze crustal stress field condition, and to describe the static and the dynamic state of the related anisotropic parameters (GAO et al, 1999). The studies indicate that the complex geological structure can result in the difference of the polarization of shear-wave splitting (Shih and Meyer, 1990; GAO et al, 1995, 1999). Because the polarization of shear-wave splitting reflects the direction of compression axis of the crust under seismic station, it can be used to study the crustal stress field characteristics (Crampin, 1978; GAO et al, 1995; Cochran et al, 2003). The seismologists in China have applied shear-wave splitting to studying the crustal anisotropy and obtained many research results (YAO et al, 1992; GAO et al, 1995, 1999; WANG et al, 1997; WANG et al, 2000; LAI et al, 2002; SHI et al, 2006; WU et al, 2007). Shear-wave splitting is sensitive to anisotropy, recent research indicates that the parameters of the shear-wave splitting can reflect the process of stress accumulation and the stress release before

\footnotetext{
* Received 2007-12-14; accepted in revised form 2008-02-25.

Foundation item: Essence Scientific Research Item of Institute of Earthquake Science, China Earthquake Administration.

^Author for correspondence: ahangyongjiu@163.com
} 
earthquakes, and also can be used to predict the earthquake (Gao and Crampin, 2004).

Sichuan area locates east to Tibetan Plateau. It is at the middle segment of South-North Earthquake Belt. The earthquake structure of Sichuan area is complex, and it is one of the strongest seismically active areas of Chinese mainland. The seismic activity is extremely frequent. Strong earthquake activity level ranks the fourth after Xizang, Yunnan and Xinjiang. From the 1970s, there were three earthquakes with $M 7,15$ earthquakes with $M 6,62$ earthquakes with $M 5$ in Sichuan area. CHEN et al $(1995,1996)$ researched anisotropy of shear-wave using the aftershock data of Shimian earthquake with M5.2 in 1989 and the Sn wave data of regional telemetry station from July, 1994 to March, 1995. This paper will use the digital seismic data since 2000 to preliminarily study the anisotropy of Sichuan area, and analyze the stress distribution characteristics in this region and its relation with the fault.

\section{Background of geological structure and station distribution}

There are mainly three fault zones in Sichuan area, which are Longmenshan, Xianshuihe and Anninghe-Zemuhe fault zones (DENG et al, 2002). There is abrupt variation in crustal thickness or gravity gradient belt along NNE in deep Longmenshan fault zone, the shallow part is made up of many faults and intense structure deformation belts, it is active block boundary zone of eastern margin of Tibetan Plateau, many faults have strong Holocene activity. Xianshuihe fault zone and Garzê-Yushu fault zone, northwest to Xianshuihe fault, are northeast boundary of eastern Tibetan block and the northern boundary of Sichuan-Yunnan block, which has strong activity in Late Quaternary. There is NNE secondary active strike-slip reverse fault, for example Yulongxi fault and so on. The eastern boundary of Sichuan-Yunnan is composed of the near S-N Anninghe fault zone, NNW Zemuhe fault zone and near S-N Daliangshan fault zone.

The principal compressive stress of Sichuan area is relatively complex, different area exists obvious difference (KAN, 1980; KAN et al, 1977, 1983; XU et al, 1987; CUI and XIE, 1999; CUI et al, 2006). According to observation results from GPS (ZHANG et al, 2003; QIAO et al, 2004), Sichuan area could be divided into three sub-blocks according to the strain and stress characteristics, which include Yajiang block, Sichuan-Qinghai block and Central Sichuan block (CHENG et $a l, 2003,2006)$. The action of Assam area which is influenced by the Indian Plate and Eurasian Plate results in inhomogeneous movement of each block, and because in the stress field of crust (stress concentration) (MA, 1980; DING, 1991), different positions have different stress conditions, there exist obvious difference in the movement forms of each block and the boundary belt.

Focal mechanism solution (PU and GUO, 1993; CHENG et al, 2006; ZHONG and CHENG, 2006) shows that the dominant direction of compression axis of Yajiang block is ESE or WNW, $P$-axis is close to horizontal, $P$-axis azimuth concentrates relatively between $0^{\circ}$ and $20^{\circ}$. The dominant direction of compression axis of Sichuan-Qinghai block is between $70^{\circ}$ and $130^{\circ}, P$-axis action presents low angle, $P$-axis azimuth concentrates relatively between $0^{\circ}$ and $30^{\circ}$. The movement velocity of Sichuan-Qinghai block is smaller than Yajiang block, Sichuan-Qinghai block lags behind Yajiang block, Xianshuihe fault between two blocks presents strong left-lateral movement. Except for strike-slip style earthquake in Sichuan-Qinghai block, the dip-slip style earthquake also accounts for a certain proportion. The distribution of the dominant direction of compression axis about small earthquake is SE or NE.

Chengdu Digital Seismic Network was put in operation from May, 2000. At first; it is made up of 14 substations, such as Guodashan (GDS), Mengdingshan (MDS), Xianjiaping (XJP) and so 
on. Along with the "tenth five-year" programs for the station extension, the number of substations increases to 19 in November, 2006. The process of adjustment is approximately that: putting Litang station on working in October, 2003; Chengdu station was increased in March, 2004; Huayingshan station was adopted instead of Laozhaizi station in January, 2006; on February 14, 2006 the substation number was increased from 16 to 19, the Litang station and Chengdu station were cancelled, simultaneously putting Xiangcheng station, Baiyu station, Batang station, Rangtang station, Songpan station in operation (Figure 1). There are six substations with the broadband digital seismograph, other substation with short period digital seismograph; the normalization amplitude-frequency response value of broadband digital seismograph is approximately equal to 1 between 0.05 and $40 \mathrm{~Hz}$, the normalization amplitude-frequency response value of short period digital seismograph is approximately equal to 1 between 1 and $20 \mathrm{~Hz}$; the dynamic range of the 16 bit substation system is more than $90 \mathrm{~dB}$, the dynamic range of the 24 bit substation system is more than $140 \mathrm{~dB}$, the actual dynamic range of the 16 bit is mostly more than $80 \mathrm{~dB}$, the actual dynamic range of the 24 bit is more than $110 \mathrm{~dB}$; the smallest measurable ground motion signal is $2.0 \times 10^{-8} \mathrm{~m} / \mathrm{s}$, the time error is smaller than $1 \mathrm{~ms}$.

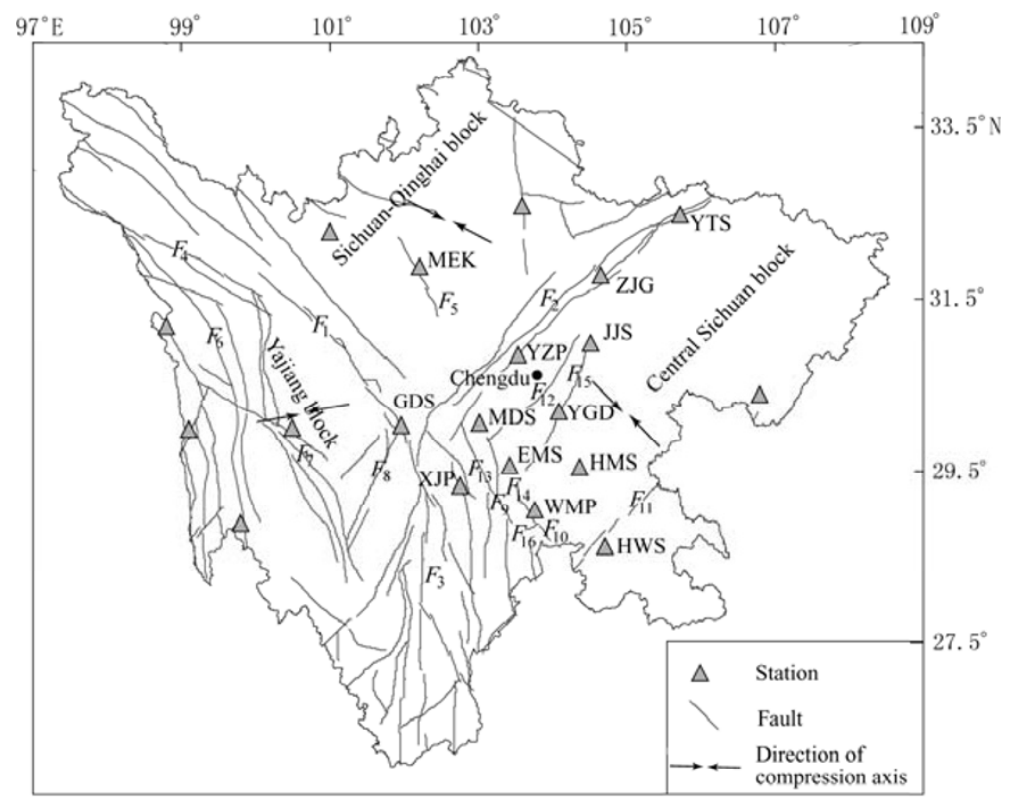

Figure 1 Distribution of seismic station and fault in Sichuan

Six stations which are not labeled in figure have not earthquake record used in this research. $F_{1}$ : Xianshuihe fault; $F_{2}$ : Longmenshan fault; $F_{3}$ : Anninghe-Zemuhe fault; $F_{4}$ : Garzê-Yushu fault; $F_{5}$ : Maerkang fault; $F_{6}$ : Jinshajiang fault; $F_{7}$ : Litang-Yajiang fault; $F_{8}$ : Yulongxi fault; $F_{9}$ : Ebian fault; $F_{10}$ : Zhongdu fault; $F_{11}$ : Huayingshan fault; $F_{12}$ : Xinjin-Chengdu-Deyang fault; $F_{13}$ : Tianquan-Rongjing fault; $F_{14}$ : Lidain fault; $F_{15}$ : Longquanshanxiyuan fault; $F_{16}$ : Dianlanba fault

\section{Method and data processing}

When the seismic wave travels in the anisotropic medium, the shear-wave split into fast shear-wave and slow shear-wave with different velocity, both of them have nearly-orthogonal polarizations character. In the crustral rock, cracks are erect and parallel, namely EDA cracks (Crampin and Atkinson, 1985). The EDA crack structure is anisotropic, and the shear-wave would 
split into fast and slow shear-waves when it travels in this kind of structure. The polarization direction of fast shear-wave is consistent with the direction of the crack caused by stress, that is, the polarization direction of fast shear-wave is in accordance with the direction of local maximum principal compressive stress. The time delay of slow shear-wave reflects the anisotropic degree of crust, which is influenced by the physical features of the crack and the character of the fluid-saturated. When shear-wave encounters free surface, reflection will be occurred if the incident angle is greater than the critical angle, which is the limited range of the shear-wave window. The critical angle is about $35^{\circ}$ in Poisson medium, because the influence of deposit of crustal surface, according to the single-layer medium computation, we choose earthquake radial with the incident angle smaller than $45^{\circ}$, which could satisfy the request of the shear-wave window (Crampin, 2005).

This study adopts the shear-wave splitting system analytic method (SAM) (GAO et al, 2004b). SAM method is proposed based on the correlation function. It includes three contents of the calculation of correlation function, elimination of time-delay and analysis of polarization, and has character of self-examination (GAO and ZHENG, 1994; GAO et al, 2004b). The new software system of SAM method has upgraded to SAM $(2007)^{\circledR}$, the specific calculation principle and analysis method can refer to other articles (e.g., GAO and ZHENG, 1994; GAO et al, 1995; 1996; 1999; SHI et $a l^{2}, 2006$; WU et al, 2007).

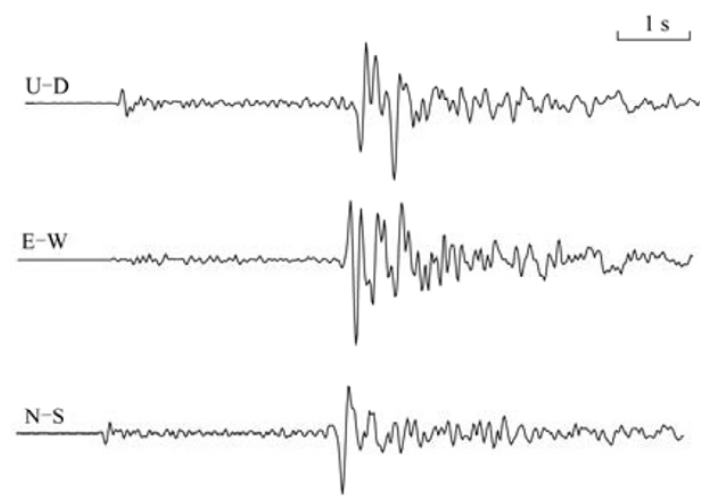

Figure 2 Waveform of the $M_{\mathrm{L}} 2.2$ earthquake on January 10, 2005 at station MEK
Before the calculation of correlation function, elimination of time-delay and analysis of polarization, the seismic data needs pretreatment, for example filtering processing and so on. According to amplitude-frequency characteristic of the seismographs and actual condition of the $\mathrm{S}$ wave splitting in Sichuan area, all of the seismic data are adopted band-pass filtering, the range of frequencies are 1 20 $\mathrm{Hz}$. Maerkang (MEK) station preliminarily data filtered waves are shown in Figure 2. The recorded earthquake with $M_{\mathrm{L}} 2.2$ occurred on January 10,2005 . The epicenter is located at $\left(31.95^{\circ} \mathrm{N}, 102.22^{\circ} \mathrm{E}\right)$, the depth of

$11 \mathrm{~km}$ and epicenter distance is $6.4 \mathrm{~km}$, according to the velocity structure model of monolayer medium, and the incident angle is $30.5^{\circ}$.

Analysis technique and process of shear-wave splitting are shown in Figure 3. Figure 3a shows that the tracks of the particle movement of two horizontal shear-waves, that is, shear-wave polarization diagram. Because shear-waves have split, the track of the particle movement is non-linear for the two components of the horizontal direction. If the angle of fast shear-wave polarization direction is $\alpha$, shear-wave is rotated clockwise angle $\alpha$ to obtain the fast and slow shear waves, then the slow shear-wave is moved up to eliminate the effect of the time delay and the polarization diagram is obtained (Figure 3b), thus, polarization figure of two shear-waves must show nearly-linear polarization. If there is something wrong with the polarization direction of the fast

\footnotetext{
${ }^{(1)}$ GAO Yuan, SHI Yu-tao, LIU Xi-qiang, et al. 2008. Personal communication.

(3) SHI Yu-tao, GAO Yuan, WU Jing, et al. 2008. Personal communication.
} 
shear-wave, no fast and slow shear-wave will be gotten because two waveforms are mixed together. If the time delay is not correctly calculated, there will be non-linear feature in the polarization. If there is some inconsistency in the result, the waveform and the polarization will be modified. This is the advantage of SAM method (GAO et al, 1995; 1996; 2004a, b).
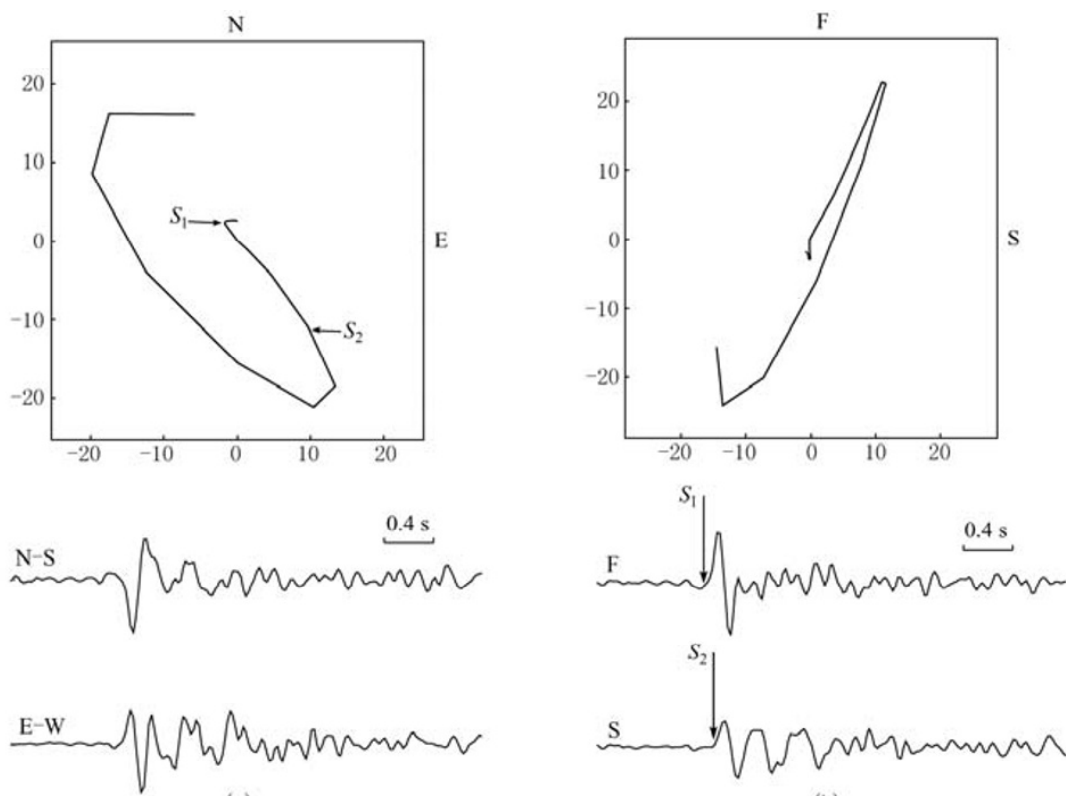

(b)

Figure 3 Analysis of shear-wave splitting

(a) Upper: Particle motion; Lower: Original shear-wave record, N-S represents North-South component and E-W represents East-West component. (b) Upper: Particle motion after the correction of wave record with the time delay between the fast and slow $\mathrm{S}$ wave; Lower: $\mathrm{F}$ represents fast $\mathrm{S}$ wave and $\mathrm{S}$ represents slow $\mathrm{S}$ wave $S_{1}$ and $S_{2}$ mark the onset of fast and slow shear-wave

\section{Results}

According to earthquake catalogue from Chengdu Digital Seismic Networks, we collect and sort 887 earthquakes with depth recorded and $M \geq 2.0$ from May, 2000 to December, 2006. As mentioned above, the critical incident angle of shear wave window theory is $35^{\circ}$, but when ray is close to surface, it is influenced by the low velocity layer or the deposit, the track of ray will tend to vertical direction gradually. According to the Sichuan area's velocity structure (ZHAO and ZHANG, 1987) and the shallow layer exploration velocity results of the petroleum department, the velocity structure is given that thickness in Sichuan area respectively is $1 \mathrm{~km}, 5 \mathrm{~km}, 20 \mathrm{~km}$, the speed is $3.7 \mathrm{~km} / \mathrm{s}, 4.3 \mathrm{~km} / \mathrm{s}$ and $5.9 \mathrm{~km} / \mathrm{s}$. For an earthquake with the depth of $12 \mathrm{~km}$, the epicentral distance is $19.5 \mathrm{~km}$, according to single-layer uniform model; the incident angle is $58.5^{\circ}$ by calculation, but according to the hierarchical model, the actual incident angle is $35.3^{\circ}$. In this research we take the incident angle $58^{\circ}$ as the shear wave window using the single-layer model, according to velocity model, the using data are all in shear-wave window. According to constraint of the shear-wave window, 152 effective earthquake records are obtained in shear-wave window. We can choose the better waveform records from 152 effective earthquake records, finally, 89 earthquake records are selected to calculate the shear-wave splitting, the shear-wave splitting results of 13 stations are obtained, there are eight stations with more than three event records (Figure 4). 

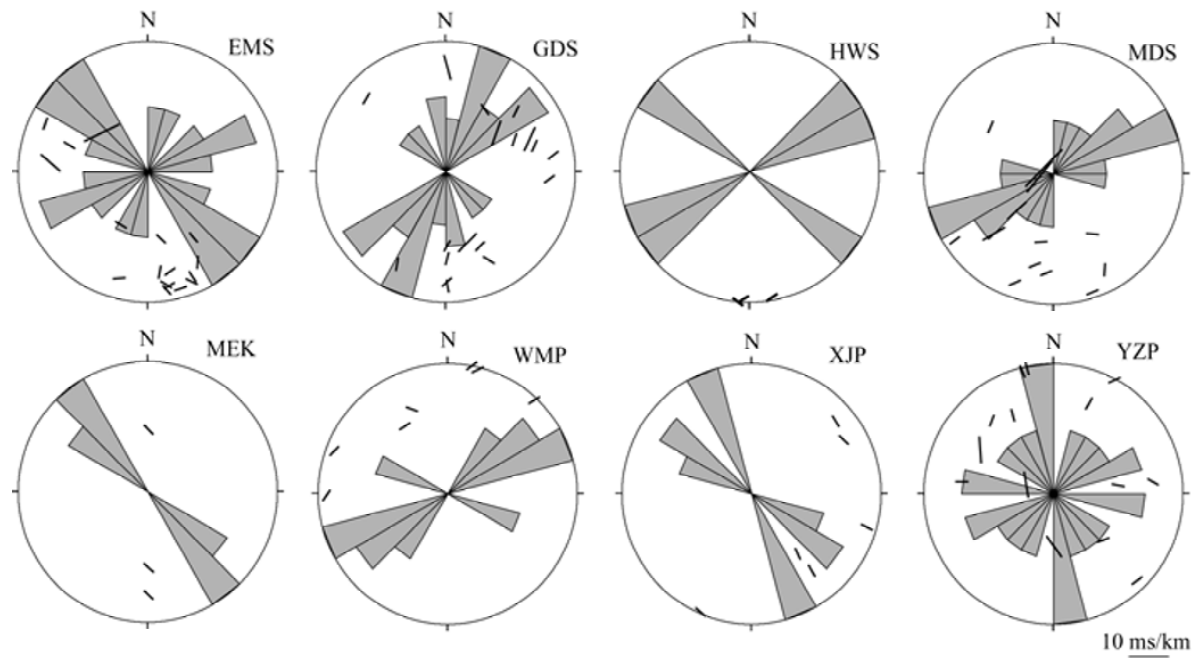

Figure 4 Lower hemispherical project and equal-area rose diagram of fast shear-wave polarizations

The directions of the short lines in the figure is each polarization of the fast shear-wave recorded by corresponding station, and the length represent the delay time level of slow shear-wave

Because the polarization direction of the fast shear-wave is inconsistent with those from different seismic events received in the same station, therefore, we adopt the range of $R$ value as between 0 and 1.0, its size reflects centralized degree of the polarization direction of the fast shear-wave, the closer to 1.0, the more centralized the direction of polarization of the fast shear-wave is (Davis, 1986; Mardia and Jupp, 2000; MA et al, 2007)

$$
\begin{gathered}
\bar{\theta}=\tan ^{-1}\left(\frac{\sum_{i=1}^{n} \sin \theta_{i}}{\sum_{i=1}^{n} \cos \theta_{i}}\right) \\
R=\frac{1}{n} \sqrt{\left(\sum_{i=1}^{n} \sin \theta_{i}\right)^{2}+\left(\sum_{i=1}^{n} \cos \theta_{i}\right)^{2}}
\end{gathered}
$$

where, $n$ represents seismic event number which is recorded by station, $\theta_{i}$ represents the polarization direction of the fast shear-wave from each seismic event. The average polarization direction of the fast shear-waves is $83.1^{\circ} \pm 49.1^{\circ}$ using 89 shear-waves splitting events from Chengdu Seismic Network, value $R$ is 0.68 , the average time delay of the slow shear-wave is $3.92 \pm 2.72 \mathrm{~ms} / \mathrm{km}$, the computed results of partial stations are listed in Table 1 (only the results of stations with more than three events of shear-wave splitting).

The polarization direction of the fast shear-wave is in accordance with the direction of the local compression axis; they are controlled by the local stress field and related to fault distribution and geological structure. Figure 5 shows that the polarizations of the fast shear-wave splitting of eight stations and its relation with stress field and fault distribution in Sichuan area. It can be seen from the Figure 5 that there are three stations (EMS, MEK, XJP) with the dominant directions of polarizations of fast shear-wave near NW or NNW direction, one station (YZP) with the polarization of fast shear-wave of NS, four stations (GDS, MDS, WMP, HWS) with the polarization of fast 
shear-wave of NE or ENE. Except for YZP station, the dominant polarization direction of the fast shear-wave is consistent with the direction of regional stress field or fault at other stations. We will carry on the more detailed discussion.

Table 1 Parameter of shear-wave splitting and regional station from Chengdu seismic network

\begin{tabular}{lccccc}
\hline Name of station & Code & Number & $\bar{\theta} /{ }^{\circ}$ & $R$ & $\overline{\Delta t} / \mathrm{ms} / \mathrm{km} \cdot \mathrm{s}^{-1}$ \\
\hline Guodashan & GDS & 18 & $47.4 \pm 53.0$ & 0.68 & $4.11 \pm 2.87$ \\
Emeishan & EMS & 17 & $93.7 \pm 47.8$ & 0.71 & $3.85 \pm 2.56$ \\
Mengdingshan & MDS & 14 & $56.5 \pm 23.3$ & 0.93 & $5.72 \pm 4.24$ \\
Youzhaping & YZP & 14 & $111.3 \pm 54.6$ & 0.62 & $3.73 \pm 1.95$ \\
Wumaping & WMP & 7 & $60.7 \pm 23.6$ & 0.93 & $2.53 \pm 0.93$ \\
Xianjiaping & XJP & 6 & $138.4 \pm 15.7$ & 0.97 & $2.59 \pm 1.34$ \\
Hanwangshan & HWS & 3 & $78.7 \pm 39.1$ & 0.85 & $2.48 \pm 0.47$ \\
Maerkang & MEK & 3 & $133.3 \pm 02.9$ & 0.99 & $1.96 \pm 1.03$ \\
\hline
\end{tabular}

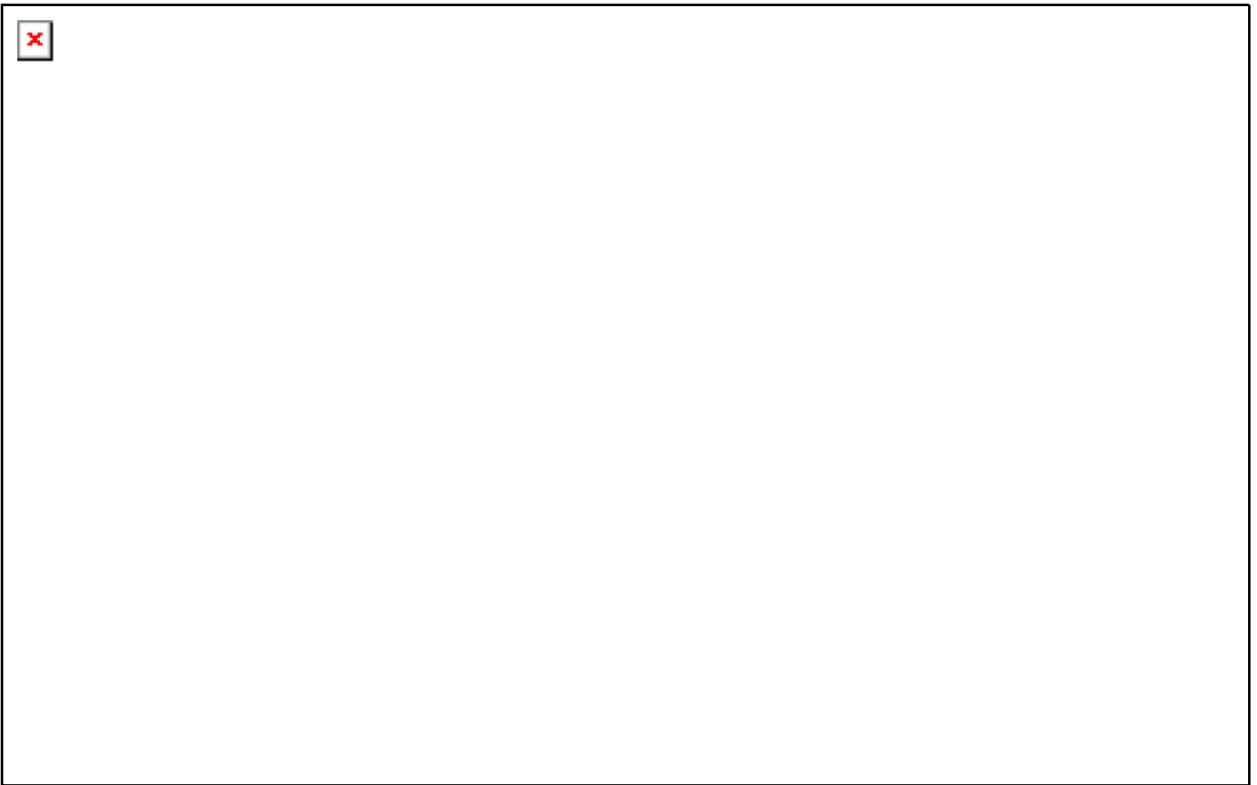

Figure 5 Polarization of fast shear-wave in Sichuan area Each fault is the same as that in Figure 1

\section{Analysis and discussion}

The shear-wave velocity structure of Sichuan area has very strong lateral heterogeneity (ZHAO et al, 1987). The complex fault distribution of Sichuan area (Figure 1) reflects complex structure in this area, the complex geologic structure results in regional character of crustal stress field in Sichuan area. The result of this research shows this kind of complex stress field.

For the reliability of results, this article only analyzes the results of stations with more than three effective data in the shear-wave window, which are eight stations.

Synthesis research result indicates Qianning-Huiyuansi detaching basin divide Xianshuihe fault into two segments, which is northwest and southeast segment. The structure of northwest segment is relatively single, it has a main fault; the structure of southeast segment is relatively 
complex, it is made up of four branch faults (WEN, 2000). Guodashan (GDS) station locates in intersection of the south end of NW Yalahe located in the middle and south of Xianshuihe fault, Zheduotang fault and north end of NNE Yulongxi fault. The polarization of the fast shear-wave is NNE or NE. Since 1970s, focal mechanism result of moderate earthquake occurred at Xianshuihe fault has shown that the direction of compression axis of this fault zone is ENE or NE (LIN et al, 1986). For example $P$-axis azimuth of Luhuo earthquake with $M 7.6$ in 1973 is $250^{\circ}, P$-axis azimuth of Daofu earthquake with $M 6.9$ in 1981 is $84^{\circ}, P$-axis azimuth of Garzê earthquake with M6.0 in 1982 is $50^{\circ}$. The dominant direction of the fast shear-wave is not consistent with the direction of the NW Xianshuihe main fault, and is different from the direction of compression axis of Xianshuihe main fault; it is in accordance with the strike of ENE Yulongxi fault. In addition, according to the fine location result of small earthquake in the western Sichuan area (ZHU et al, 2005), analysis shows Moxi zone exists NE hidden fault. The analysis result of the fast shear-wave polarization of this station has supported this conclusion to a certain extent.

Measurement result of ground stress caused by hydraulic fracturing shows that the maximum horizontal compression axis of Longmenshan fault has difference at different segments; the direction is NW-SE (AN et al, 2004). Mengdingshan (MDS), Youzhaping (YZP), Zhongjiagou (ZJG) and Yingtaishan (YTS) stations locate respectively at Longmenshan fault and near different segments, because the earthquake record number of ZJG and YTS stations is less than 3, this article does not discuss them. The MDS station locates at the juncture of the south end of Longmenshan fault, NE Xinjin-Chengdu-Deyang fault, NNW Tianquan-Rongjing fault and Ebian fault, the dominant direction of polarization of the fast shear-wave is $\mathrm{NE}$, which is approximately vertical to the direction of compression axis, consistent with the strike of Xinjin-Chengdu-Deyang fault, relatively consistent with the strike of Longmenshan fault, and is independent of the strike of Tianquan-Tongjing and Ebian fault. A possible explanation is that the earthquake used to analyze the fast shear-wave polarization is even closer to the Xinjin-Chengdu-Deyang fault; the strikes of Longmenshan fault and Xinjin-Chengdu-Deyang fault are relatively close.

Youzhaping (YZP) station is located in the middle segment of Longmenshan fault, transition zone from basin to plateau, the dominant direction of polarization of the fast shear-wave is near $\mathrm{N}-\mathrm{S}$, which is obvious different from the direction of local compression axis, the direction of principal compressive strain from GPS (ZHANG et al, 2003, 2005) and the strike of Longmenshan fault.

This result makes it difficult to explain. But further analysis will make it clear that (1) the altitude of this station is $608 \mathrm{~m}$, which is close to that of the edge of basin, the stylobate is influenced by the deposition of basin edge, which results in relatively complex earthquake records of this station, there are 12 records which appear "conversion waveform" in 14 records of the earthquake (YAO et al, 1992), which creates the relatively big disturbance to our analysis; (2) the azimuths of earthquake which the polarization of the fast shear-wave is near NS are all more than $280^{\circ}$ or less than $30^{\circ}$, that is, these earthquakes are all located in the northwestern of these stations, and the depth of source is below $10 \mathrm{~km}$ (Table 2), therefore we conclude that there exist complicated structure below some depth in the region (ZHU et al, 2005), such as NS miniature hidden fault. For confirming the accuracy of the latter extrapolation, we need to add more data, and provide more reliable observation evidence.

Emeishan (EMS) station locates at the juncture of east side of Ebian fault, southwest end of Longquanshan fault and northwest end of Lidian fault, the dominant direction of polarization of the fast shear-wave of this station is NW, it is consistent with the direction of local compression 
axis, the direction of principal compressive strain from GPS (ZHANG et al, 2003, 2005) and the strikes of Ebian fault, Lidian fault, simultaneously the second dominant direction is close to the strike of Longquanshanxiyuan fault.

Table 2 Polarization of fast shear-wave and parameter of events is recorded at YZP station

\begin{tabular}{|c|c|c|c|c|c|c|c|}
\hline No. of events & $\begin{array}{c}\text { Date } \\
\text { a-mo-d }\end{array}$ & $\begin{array}{l}\text { Origin time } \\
\qquad \begin{array}{c}\text { (BT) } \\
\text { h:min }\end{array}\end{array}$ & Depth/km & $\begin{array}{l}\text { Magnitude } \\
M_{\mathrm{L}}\end{array}$ & Azimuth ${ }^{\circ}$ & $\begin{array}{c}\text { Epicentral } \\
\text { distance } \\
/ \mathrm{km}\end{array}$ & $\begin{array}{c}\text { Polarization of fast } \\
\text { shear-wave/ }{ }^{\circ}\end{array}$ \\
\hline 01 & 2001-11-15 & $10: 42$ & 15.0 & 2.0 & 179.20 & 7.57 & 145.0 \\
\hline 02 & $2002-11-16$ & $20: 51$ & 13.0 & 2.0 & 28.38 & 20.33 & 165.0 \\
\hline 03 & 2003-02-11 & 07:04 & 15.0 & 2.1 & 300.83 & 13.11 & 175.0 \\
\hline 04 & $2003-02-24$ & $17: 59$ & 15.0 & 2.5 & 348.18 & 22.76 & 165.0 \\
\hline 05 & 2003-03-03 & 03:05 & 11.0 & 2.0 & 332.55 & 9.79 & 165.0 \\
\hline 06 & $2003-03-22$ & $12: 57$ & 15.0 & 2.4 & 288.34 & 4.00 & 170.0 \\
\hline 07 & 2003-05-09 & $22: 44$ & 15.0 & 2.2 & 82.71 & 9.65 & 100.0 \\
\hline 08 & 2003-09-19 & $16: 10$ & 10.0 & 2.8 & 83.36 & 10.58 & 120.0 \\
\hline 09 & 2003-09-26 & $12: 31$ & 10.0 & 2.3 & 277.45 & 9.59 & 90.0 \\
\hline 10 & 2004-01-10 & $09: 22$ & 10.0 & 2.5 & 345.67 & 14.91 & 155.0 \\
\hline 11 & 2004-04-09 & $17: 20$ & 22.0 & 2.1 & 319.70 & 21.95 & 180.0 \\
\hline 12 & $2004-08-24$ & $13: 02$ & 11.0 & 2.1 & 135.85 & 15.07 & 55.0 \\
\hline 13 & 2004-10-01 & $13: 20$ & 12.0 & 2.1 & 132.82 & 7.87 & 70.0 \\
\hline 14 & 2005-09-10 & $14: 19$ & 13.0 & 3.0 & 21.62 & 13.21 & 30.0 \\
\hline
\end{tabular}
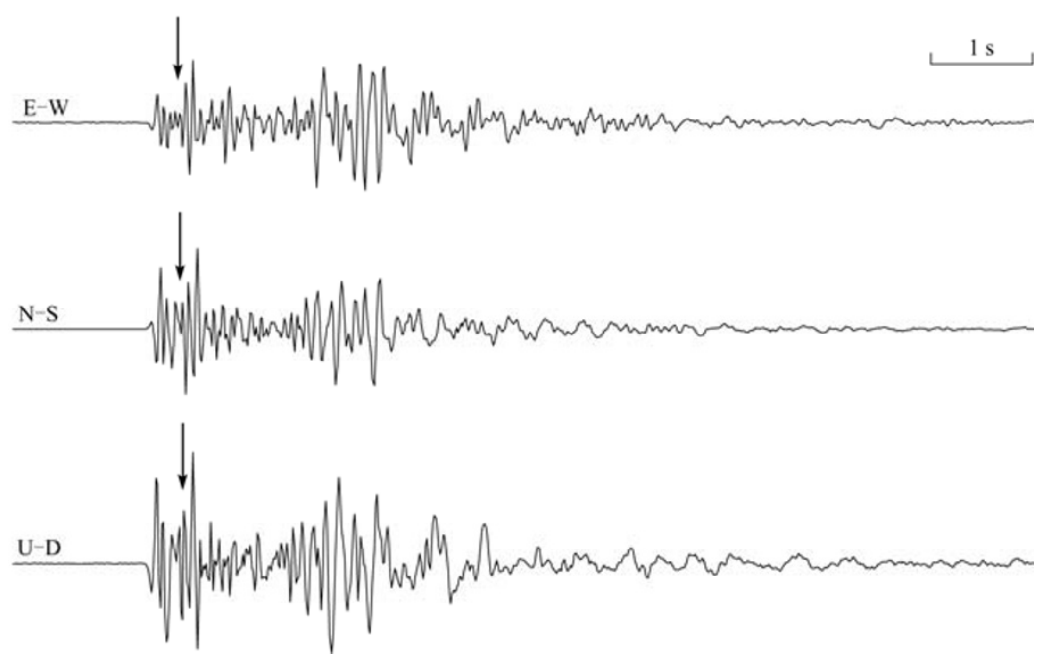

Figure 6 Earthquake waveforms recorded at YZP station on January 20, 2004 with $M_{\mathrm{L}} 2.1$ The arrow presents the seismic phase of conversion

Mabian-Yanjin fault contains many secondary faults, the strike of each secondary fault and other character exist the difference, and the strike of most secondary faults are all NNW, for example Lidian fault, Zhongdu fault. It also has ENE fault, for instance Dianlanba fault. Wumaping (WMP) station locates at the juncture of Lidian fault, Zhongdu fault and Dianlanba fault, the dominant direction of polarization of the fast shear-wave of this station is ENE, which is approximately vertical to the direction of local principal compression axis and GPS observation results, and is consistent with the strike of Dianlanba fault. A possible explanation is that, there is ENE Dianlanba fault across discontinuity of WNW Lidian fault and Zhongdu fault, the result of shear-wave splitting of this station is mainly controlled by ENE Dianlanba fault. 
Huayingshan fault zone locates in the southeast of Sichuan basin, which is compresso-shear fault-fold belt. The seismic activity appears mainly as double earthquake and earthquake swarms in this fault-fold belt and neighboring area (LIU, 2000). Hanwangshan (HWS) station locates in the southwest segment of Huayingshan fault; the analysis result of shear-wave splitting of this station shows dual influences of stress field and fault strike. The dominant direction of polarization of the fast shear-wave from one record is NE, which is consistent with the strike of Huayingshan fault; the polarization direction of the fast shear-wave using other record is NW, which is in accordance with the direction of local principal compression axis. We need accumulate the data of HWS station to make further analysis.

Maerkang fault locates in the Sichuan-Qinghai block, it controls NW fault valley, such as Fubianhe. The synthesis result of focal mechanism from Xiaojin earthquake with M6.6 in 1989 shows, the principal compression axis of this fault is consistent with regional stress field (DIAO et $a l, 1995)$. Maerkang (MEK) station locates at Songgang segment of Maerkang fault, the polarization of the fast shear-wave is NW, which is in accordance with the direction of compression axis direction of Songgang segment of Maerkang fault, the direction of principal compressive strain from GPS (ZHANG et al, 2003, 2005) and the strike of Songgang segment of Maerkang fault.

Xianjiaping (XJP) station locates at the juncture of the north of Ganluo-Zhuhe fault and Baoxinchang-Fengyi fault, the strikes of two faults are consistent at the juncture, and they are all NNW. The polarization of the fast shear-wave is NW, which is consistent with the direction of

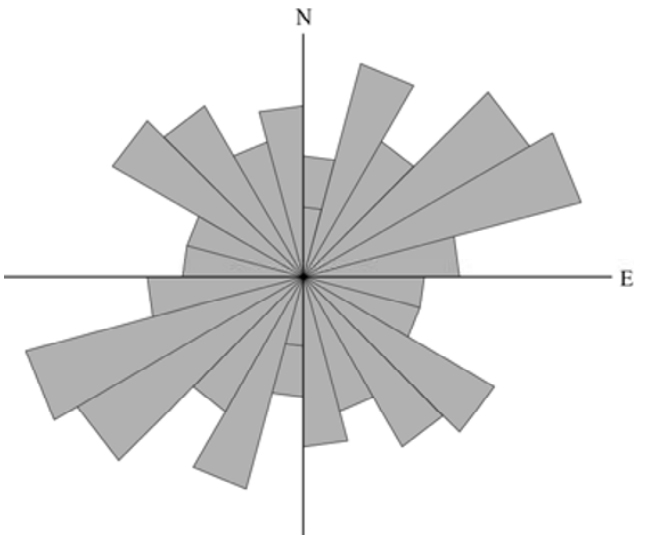

Figure 7 Project and equal-area rose diagram of fast shear-wave polarizations Results of 13 stations, including five stations with 1 2 available waveform local compression axis, the direction of principal compressive strain from GPS (ZHANG et al, 2003, 2005) and the strike of Ganluo-Zhuhe fault.

We synthesize the data of 13 stations with analysis results, the homolographic projection rose diagram of the polarization of the fast shear-wave using all records are obtained (Figure 7). Figure 7 shows two dominant directions, one is near NE direction, and it is consistent with the strike of mostly fault zone (Longmenshan fault zone and so on) in research region. The other is approximately NW direction, which is in accordance with the direction of principal compression axis in research region.

\section{Conclusions}

The Sichuan area is strongly influenced by the Eurasian Plate and the Indian Plate, the geophysical and geological characteristics of the deep, the shallow and surface are all very complex. Using the waveform data recorded by Chengdu Digital Seismic Network from May 1, 2000 to December 31, 2006 in Sichuan Province, some preliminary understandings about crustal anisotropy in Sichuan area can be obtained through research on shear-wave splitting.

The dominant direction of polarization of fast shear-wave represents the direction of compression axis of the local region (a station and neighboring) or the active fault. The study shows 
that the polarization of fast shear-wave is consistent with the direction of principal compressive strain from GPS and the direction of the local principal compression axis, and it is strongly related to the strikes of active faults. The dominant directions of polarizations of fast shear-waves at most of stations are mainly at nearly NE or NW direction in Sichuan.

The fast shear-wave polarizations of stations located at the active faults or intersection of several active faults are mostly consistent with the strikes of active faults which control the earthquake activities, and are in accordance with the regional principal compression axis, for example, GDS, EMS, XJP, WMP, MEK, HWS and MDS stations.

A complex local structure could control or influence the principal directions of polarization of fast shear-wave of station, which result in that this direction is inconsistent with the strike of the major active fault, or there is greater different from results of local principal compression axis, for example YZP station. But this phenomenon needs careful analysis, the distribution of fast shear-wave polarization not only may judge the direction of principal compression field, but may also analyze the fault activity, and promulgate the new structure relations, for instance whether to have the underlying fault and so on (SHI et al, 2006; WU et al, 2007).

Sichuan area structure is complex with many fault zones and many feeling earthquakes, at present, the station with relatively good and rich records still mainly concentrate in the edge of west and north Sichuan, northwestern Sichuan, southwestern Sichuan plateau and eastern Sichuan areas have few digital seismic data, more data are needed to conduct the further more comprehensive research work. About the influence of Xianshuihe fault zone, we also need more data to conduct the precise seismic research.

Acknowledgements The authors express their thanks to WU Jing and TAI Ling-xue for their help.

\section{References}

AN Qi-mei, DING Li-feng, WANG Hai-zhong, et al. 2004. Research of property and activity of Longmen Mountain fault zone [J]. Journal of Geodesy and Geodynamics, 24(2): 115-119 (in Chinese).

CHEN Tian-chang, FAN Jun, ZHENG Jian. 1996. Sn-wave polarization in Sichuan region [J]. Earthquake Research in Sichuan, (4): 85-90.

CHEN Tian-chang, LI Zhi-jun, ZHANG Run-sheng, et al. 1995. Temporal changes in shear wave splitting after the $M=5.2$ Shimian Earthquake of June 9, 1989 [J]. Acta Seismologica Sinica, 8(1): 65-76.

CHENG Wan-zheng, DIAO Gui-ling, LÜ Yi-pei, et al. 2003. Focal mechanisms, displacement rate and mode of motion of the Sichuan-Yunnan block [J]. Seismology and Geology, 25(1): 71-87 (in Chinese).

CHENG Wan-zheng, RUAN Xiang, ZHANG Yong-jiu. 2006. Types of focal mechanism solutions and parameter consistency of the sub-blocks in Sichuan and Yunnan provinces [J]. Acta Seismologica Sinica, 19(6): 605-619.

Cochran E S, Vidale J E, Li Y G. 2003. Near-fault anisotropy following the Hector Mine earthquake [J]. J Geophys Res, 108(B9): $2436-2447$.

Crampin S and Atkinson B K. 1985. Microcracks in the Earth's crust [J]. First Break, 3(3): 16-20.

Crampin S. 1978. Seismic-wave propagation through a cracked solid: Polarization as a possible dilatancy diagnostic [J]. Geophys $J R$ Astron Soc, 53: 467-496.

Crampin S. 2005. A review of shear-wave splitting in the compliant crack-critical anisotropic earth [J]. Wave Motion, 41: 59-77.

CUI Xiao-feng and XIE Fu-ren. 1999. Preliminary research to determine stress districts from focal mechanism solution in Southwest China and its adjacent area [J]. Acta Seismologica Sinica, 12(5): 562-572.

CUI Xiao-feng, XIE Fu-ren, ZHANG Hong-yan. 2006. Recent tectonic stress field zoning in Sichuan-Yunnan region and its dynamic interest [J]. Acta Seismologica Sinica, 19(5): 485-496.

Davis J C.1986. Statistics and Data Analysis in Geology [M]. Hoboken N J: John Wiley: 649.

DENG Qi-dong, ZHANG Pei-zhen, RAN Yong-kang. 2002. Basic characteristics of active tectonics of China [J]. Science in China (Series $D):$ 32(12): $1020-1030$ (in Chinese).

DIAO Gui-ling, CHENG Wan-zheng, LI Gui-fang, et al. 1995. The stress field in source region of the Xiaojin earthquake sequence in Sichuan [J]. Earthquake Research in China, 11(2): 168-173.

DING Guo-yu. 1991. Lithospheric Dynamics of China [M]. Beijing: Seismological Press: 154-178 (in Chinese).

Gao Y and Crampin S. 2004. Observations of stress relaxation before earthquakes [J]. Geophys J Int, 157(2): 578-582.

GAO Yuan and ZHENG Si-hua. 1994. On shear wave splitting in Tangshan region (II): Correlation function analysis method [J]. Earthquake Research in China, 10(Suppl.): 11-21 (in Chinese). 
GAO Yuan, LIANG Wei, DING Xiang, et al. 2004a. Variational characteristics of shear-wave splitting on the 2001 Shidian earthquake in Yunnan, China [J]. Acta Seismologica Sinica, 17(6): 635-641.

GAO Yuan, LIU Xi-qiang, LIANG Wei, et al. 2004b. Systematic analysis method of shear-wave splitting: SAM software system [J]. Earthquake Research in China, 20(1): 101-107 (in Chinese).

GAO Yuan, ZHENG Si-hua, SUN Yong. 1995. Crack-induced anisotropy in the crust from shear wave splitting observed in Tangshan region, North China [J]. Acta Seismologica Sinica, 8(3): 351-364.

GAO Yuan, ZHENG Si-hua, WANG Pei-de. 1996. Shear wave splitting study on small earthquake swarm of 1992 in Dongfang of Hainan, China [J]. Chinese J Geophys, 39(2): 221-232 (in Chinese).

GAO Yuan, ZHENG Si-hua, ZHOU Hui-lan. 1999. Polarization patterns of fast shear wave in Tangshan region and their variations [J]. Chinese J Geophys, 42(2): 228-232 (in Chinese).

KAN Rong-ju, WANG Shao-jin, HUANG Kun, et al. 1983. Modern stress field and relative motion of intraplate block in southwestern China [J]. Seismology and Geology, 5(2): 79-90.

KAN Rong-ju, ZHANG Si-lu, YAN Feng-tong. 1977. Study on the current tectonic stress field and the characteristics of current tectonic activity in southwest China [J]. Chinese J Geophys, 20(2): 96-109 (in Chinese).

KAN Rong-ju. 1980. The current tectonic stress field in southwest China and the inner strong seismic activity of block [J]. Journal of Seismological Research, 3(3): 45-59 (in Chinese).

LAI Yuan-gen, LIU Qi-yuan, CHEN Jiu-hui. 2002. Features of the S-wave splitting and stress field in the Xinjiang Jiashi strong earthquake region [J]. Chinese J Geophys, 45(1): 83-93 (in Chinese).

LIN Bang-hui, CHEN Tian-chang, PU Xiao-hong, et al. 1986. Rupture processes of strong earthquakes on Xianshuihe fault belt and seismic activity [J]. Acta Seismologica Sinica, 8(1): 1-20 (in Chinese).

LIU Cheng-ming. 2000. New seismic characteristics of Huayingshan fault fold belt and inner reason discussion [J]. Earthquake Research in Sichuan, (4): 29-33 (in Chinese).

MA Hong-sheng, ZHANG Guo-min, LIU Jie, et al. 2007. Coupling characteristics of stress and strain at different layers of different sub-regions in Yunnan and its adjacent areas [J]. Acta Seismologica Sinica, 20(2): 133-146.

MA Zong-jin. 1980. The multi-spot stress field and seismic activity of North China [J]. Seismology and Geology, 2(1): 39-48 (in Chinese).

Mardia K V and Jupp P E. 2000. Directional Statistics [M]. Hoboken N J: John Wiley: 429.

PU Xiao-hong and GUO Hui-lan. 1993. The character of current tectonic stress field interspace distribution and determination of latency hypocenter in Sichuan [J]. Northwestern Seismological Journal, 15(2): $97-99$ (in Chinese).

QIAO Xue-jun, WANG Qi, DU Rui-li. 1993. Characteristics of current crustal deformation of active blocks in the Sichuan-Yunnan region [J]. Chinese J Geophys, 47(5): 805-811

SHI Yu-tao, GAO Yuan, WU Jing, et al. 2006. Seismic anisotropy in the crust in Yunnan of China: Polarizations of fast shear-waves [J]. Acta Seismologica Sinica, 19(6): 620-632.

Shih X R and Meyer R P. 1990. Observation of shear wave splitting from nature events: South Moat of long Valley Caldera, California, June 29 to August 12, 1982 [J]. J Geophys Res, 95: 11 179-11 196.

WANG Chun-yong, DING Zhi-feng, CHEN Xue-bo, et al. 1997. The S-wave splitting and medium anisotropy of lithosphere in Dabieshan zone [J]. Chinese Science Bulletin, 42(23): 2 539-2 542.

WANG Pei-de, Klinge K, Kruger F, et al. 2000. Variation of shear-wave splitting in earthquake clusters with very similar waveforms [J]. Acta Seismologica Sinica, 13(5): 536-543.

WEN Xue-ze. 2000. Character of rupture segmentation of the Xianshuihe-Zemuhe fault zone, western Sichuan [J]. Seismology and Geology, 22(3): 239-249 (in Chinese).

WU Jing, GAO Yuan, CHEN Yun-tai, et al. 2007. Seismic anisotropy in the northwestern capital area of China [J]. Chinese J Geophys, 50(1): 209-220 (in Chinese).

XU Zhong-huai, WANG Su-yun, HUANG Yu-rui, et al. 1987. Direcions of mean stress axes in southwestern China deduced from microeartquake data [J]. Chinese J Geophys, 30(5): 476-486 (in Chinese).

YAO Chen, WANG Pei-de, CHEN Yun-tai. 1992. Shear-wave polarization and upper crustal crack anisotropy in Lulong [J]. Chinese $J$ Geophys, 35(3): 305-315 (in Chinese).

ZHANG Xi, JIANG Zai-sen, WANG Qi, et al. 2003. Recent crust motion features in Sichuan-Yunnan and their relationship to strong earthquake [J]. Journal of Geodesy and Geodynamics, 23(3): 35-41 (in Chinese).

ZHANG Xiao-liang, JIANG Zai-sen, WANG Shuang-Xu, et al. 2005. Preliminary research on present horizontal block motion and deformation features in Sichuan-Yunnan area [J]. Journal of Seismological Research, 28(3): 262-267 (in Chinese).

ZHAO Zhu and ZHANG Run-sheng. 1987. Primary study of crustal and upper mantle velocity structure of Sichuan Province [J]. Acta Seismologica Sinica, 9(2): 154-166 (in Chinese).

ZHONG Ji-mao and CHENG Wan-zheng. 2006. Determination of directions of the mean stress field in Sichuan-Yunnan region from a number of focal mechanism solutions [J]. Acta Seismologica Sinica, 19(4): 359-369.

ZHU Ai-lan, XU XI-wei, ZHOU Yong-sheng, et al. 2005. Relocation of small earthquakes in western Sichuan, China and its implications for active tectonics [J]. Chinese J Geophys, 48(3): 629-636. 